\title{
3) La politique commune des visas : les luttes pour l'homogénéisation ou le maintien d'un réseau hétérogène?
}

\section{Elspeth Guild et Didier Bigo}

\section{(2) OpenEdition \\ Journals}

Édition électronique

URL : http://journals.openedition.org/conflits/930

DOI : $10.4000 /$ conflits.930

ISSN : $1777-5345$

Éditeur :

CCLS - Centre d'études sur les conflits lilberté et sécurité, L'Harmattan

Édition imprimée

Date de publication : 1 mars 2003

Pagination : 71-81

ISBN : 2-7475-4868-8

ISSN : 1157-996X

Référence électronique

Elspeth Guild et Didier Bigo, «3) La politique commune des visas : les luttes pour l'homogénéisation ou le maintien d'un réseau hétérogène? ", Cultures \& Conflits [En ligne], 49 | printemps 2003, mis en ligne le 29 septembre 2003, consulté le 30 mars 2021. URL : http://journals.openedition.org/conflits/ 930 ; DOI : https://doi.org/10.4000/conflits.930

Ce document a été généré automatiquement le 30 mars 2021

Creative Commons License 


\title{
3) La politique commune des visas : les luttes pour l'homogénéisation ou le maintien d'un réseau hétérogène
} ?

\author{
Elspeth Guild et Didier Bigo
}

3) La politique commune des visas : les luttes pour l'homogénéisation ou le maintien d'un réseau hétérogène?

Les plaquettes de présentation du visa Schengen évoquent un visa uniforme pour l'ensemble du territoire européen favorisant la libre circulation des étrangers ${ }^{1}$. Mais une lecture plus attentive de la documentation montre qu'il existe plusieurs visas, que les visas n'ont pas les mêmes effets juridiques d'un pays à l'autre, qu'il s'agit de l'espace Schengen qui n'inclut pas le Royaume-Uni et l'Irlande. Quant à l'effectivité de la libre circulation des personnes et à l'information sur les taux d' acceptation ainsi que celle sur les délais d'obtention des visas, elles ne figurent pas sur la plaquette.

Le règlement de la Commission adopté par le Conseil le 24/04/2001 et ses implications quant à l'uniformité du visa

Les querelles incessantes concernant la sévérité des contrôles aux frontières extérieures et la réalité de la suppression des contrôles aux frontières intérieures ont poussé les gouvernements à abandonner la logique intergouvernementale de Schengen et à accepter le rôle de la Commission quant à une politique commune des visas, mais uniquement si celle-ci maintenait un cap restrictif et si elle (ou le Parlement) ne pouvait revenir sur « l'acquis Schengen».

Est-ce à dire que nous avons maintenant un instrument le visa permettant de définir « un » territoire européen où les règles d'entrée seraient uniformes ? Certainement pas, d'abord par la différence de territoire entre l'Union Européenne et Schengen, ensuite parce que la Commission n'a pu imposer une réelle homogénéisation.

La proposition de règlement de la Commission - qui a débouché sur le règlement du Conseil du 24 avril 2001 et qui a permis ensuite la publication au JO des Communautés 
Européennes des instructions consulaires communes et du manuel commun ${ }^{2}$ - insiste sur les principes de fond que les Etats devraient respecter (dont celui d'une véritable absence de contrôles aux frontières intérieures et la levée des clauses de sauvegarde que la France a rendu quasi permanente dans ses relations avec les Pays-Bas; un effort accru d'harmonisation globale des politiques d'immigration; un standard reconnu et de qualité pour les demandeurs d'asile) tout en essayant de mettre en place une politique commune de visas qui remplisse véritablement le rôle de "filtre homogène, possédant des critères clairs d'attribution et de non attribution, au lieu de la loterie actuelle ${ }^{3}$. Les critères, une fois définis, étant appliqués ensuite par les Etats qui resteraient ainsi maîtres de l'application.

Dès lors, le discours actuel de la Commission est le suivant. Il faut rationaliser l'acquis Schengen et renforcer le droit communautaire non issu de Schengen. Ce n'est qu'à travers une politique commune de visa et surtout une application uniforme par les autorités consulaires que l'on pourra finalement retrouver une cohérence et une certaine unification du territoire. L'Union européenne comme un ensemble territorial aura alors les moyens techniques d'exercer à distance, via le visa uniforme, un pouvoir de contrôle qui s'ajoutera à celui des Etats sans s'y substituer ${ }^{4}$.

Pour le dire autrement, la Commission laisse aux Etats le droit d'entrée (et de sortie) des individus sur leurs territoires respectifs mais elle exerce quant à elle, ce que l'on pourrait appeler une prétention à la monopolisation de la légitimité des mécanismes d'entrée des étrangers venant de pays à risques sur le territoire Schengen.

Ainsi elle ne touche pas directement aux attributs étatiques de souveraineté via la délivrance des passeports et le contrôle aux frontières physiques, en particulier à cause des britanniques, mais surajoute son contrôle à ceux-ci, via son rôle dans l'uniformisation des pays soumis à visa et l' homogénéisation des critères d'attribution. Seulement les Etats ne cessent de remettre en cause ces deux éléments. Ils introduisent des clauses visant à ne pas faire confiance à l'examen des visas par les autres parties contractantes, et surtout ils maintiennent des critères pratiques différents d'attribution des visas en fonction de leur idéologie et de la sévérité de leur politique migratoire. Le rapport de force est donc bien réel, même si tout le monde semble vouloir plus d'unité et plus d'efficacité.

Selon les entretiens réalisés auprès de la Commission, l'établissement de la nationalité des personnes qui se présentent à la frontière est crucial pour toute la politique de gestion des flux transfrontaliers et il est préférable de l'établir le plus tôt possible en amont. "L'objectif d'un espace de liberté et de sécurité se concrétise par la suppression des contrôles sur les personnes, quelle que soit leur nationalité, aux frontières intérieures, et par des contrôles harmonisés aux frontières extérieures ainsi que par une politique commune de visas ». "La politique des visas est une priorité en termes d'efficacité, et si les visas de plus de trois mois restent soumis aux procédures nationales, les visas pour un séjour n' excédant pas trois mois doivent être harmonisés ».

On pourrait penser que l'uniformisation vient du fait que quel que soit le consulat des pays de l'Union le candidat au départ recevra le même visa lui permettant de voyager sur l'ensemble du territoire européen. Seulement le détail des instructions est plus compliqué et remet en cause l'unicité du visa Schengen, de même que la possibilité qu'il y aurait de voyager avec un tel type de visa sur tout le territoire européen.

Sur le plan juridique le visa uniforme Schengen n'est pas un visa unique pour deux raisons. C'est un visa délivré par un des Etats de l'Union et qui vaut possibilité d'entrée 
dans les autres pays de l'espace Schengen, mais qui est toujours soumis à appréciation au passage des frontières intérieures. Par ailleurs, il existe huit types de visa Schengen qui ont des conséquences juridiques différentes.

Le visa Schengen, un visa néanmoins national?

Comme le signalent les instructions consulaires communes: «Le visa uniforme est l'autorisation ou la décision, matérialisée par l'apposition d' une vignette par une Partie contractante sur un passeport, un titre de voyage ou un autre document valable permettant le franchissement des frontières. Il permet à l'étranger, soumis à l'obligation de visa, de se présenter à un poste de la frontière extérieure de la Partie contractante de délivrance ou d'une autre Partie contractante pour solliciter, selon le type de visa, le transit ou le séjour, pourvu que soient réunies les autres conditions de transit ou d'entrée. Le fait d'être en possession d'un visa uniforme ne confère pas de droit d'entrée irrévocable $»^{5}$.

En effet, parce que le système de visa Schengen est un réseau de systèmes nationaux entre les Parties contractantes (c'est-à-dire, les Etats membres), le refus d'entrée à la frontière d'une personne munie d'un visa Schengen dépend des situations.

En pratique, si le visa est octroyé par l'Etat auquel appartient le fonctionnaire, le refus d'entrée aux frontières est bien possible parce que la menace est définie par l'Etat luimême, et cela n'oppose de facto que la police à ses autorités consulaires. Sauf cas exceptionnel, il n'existe pas de litige car la police a une compétence discrétionnaire.

En revanche, si le refus d'entrée à un individu qui a un visa Schengen délivré par un autre Etat est toujours possible aux frontières intérieures, ce refus est bien plus délicat car cela fait penser que cet Etat est lui-même laxiste sur sa manière de délivrer les visas ou que l'Etat de première arrivée n'a pas fait sérieusement de contrôle. Une police nationale est alors dans la situation d'émettre un jugement sur des autorités consulaires d'un autre Etat membre ou sur les autorités de contrôle aux frontières extérieures. Il y aura donc à prendre en compte les relations diplomatiques internes des Etats de l'Union. Comment refuser un visa issu d' un consulat allemand? Inversement, certains pays du Sud se sentent mis en doute sur cette question. Ainsi, les polices aux frontières en France ou en Allemagne restent très « vigilantes » et stoppent des personnes pourvues de visas Schengen issus de consulats « douteux » - les consulats grecs au Moyen-Orient et en Afrique sont souvent cités.

Certains entretiens ont mis l'accent sur le risque d'un « visa shopping » où les candidats à l'entrée choisiraient le consulat le plus laxiste et auraient ensuite accès à tout le territoire Schengen. Mais, alors que ce thème est très développé par les fonctionnaires des guichets pour justifier leur suspicion, ceci ne tient guère compte des contraintes qui pèsent sur le candidat à l'entrée. Il ne peut demander son visa que dans le consulat du pays où il restera le plus longtemps et doit donc prouver qu'il a des raisons de se rendre dans ce pays ${ }^{6}$.

Si visa shopping il y a, cela ne peut se faire en fait qu'avec la complicité, voire la corruption de certaines autorités consulaires: cela ne peut pas être une stratégie individuelle. Par ailleurs, il est quasi impossible pour un candidat au voyage de demander un visa Schengen sans le faire depuis son pays d'origine ${ }^{7}$. Enfin toute demande de dépôt multiple est considérée comme étant la preuve d'une tentative de fraude et vaut refus de visa. Ce n'est donc pas dans n'importe quel consulat que l'on peut se rendre pour demander un visa Schengen mais simplement dans celui du pays où l'on arrive et reste le plus longtemps. Là encore, l'unicité du territoire et de la libre circulation est loin d'être démontrée. 
C'est pour éviter les tensions entre les Etats de l'Union que la coopération des agents consulaires sur place est si fortement recommandée par la Commission. Si tous participent à la décision, on limitera les divergences $d$ 'appréciation qui se ressentent ensuite lors du passage à la frontière. Le visa Schengen est alors, pour résumer, non pas un visa pour le territoire européen comme on le présente trop souvent, mais un visa sur un territoire particulier, celui de Schengen. Defacto national, ce visa ouvre des facilités pour entrer sur le territoire des autres membres de l'espace mais il n'assure pas l'entrée sur un territoire unique où régnerait la libre circulation et ne peut être émis que par quelques consulats en fonction de la demande du candidat.

Le(s) 8 (5+3) Visa(s) Schengen

Non seulement le visa Schengen n'est pas un visa pour tout le territoire européen, mais, par ailleurs, le visa Schengen dit uniforme se décompose en différents types de visas. Nos interlocuteurs ne sont pas très précis sur ce point et restent parfois contradictoires $^{8}$. On peut considérer qu'il existe huit types de visa ayant les effets d'un visa uniforme. Ils se regroupent en cinq catégories et sont traités dans quatre paragraphes de l'instruction consulaire (art 2.1.1 à 2.1.4) qui définissent au sens strict le visa uniforme. Il faut encore y ajouter trois autres types de visas nationaux qui ont des liens avec les visas uniformes Schengen (art 2.2 à 2.4) ${ }^{9}$.

Les cinq catégories sont les suivantes:

Visa de transit aéroportuaire, visa autorisant un étranger qui se rend d'un Etat tiers vers un autre Etat tiers à traverser le territoire des parties contractantes dit visa de transit, visa de court séjour ou de voyage, visa à entrées multiples, et visa collectif.

Comme le décrit l'instruction consulaire, les visas sont définis ainsi :

«2.1.1 Visa de transit aéroportuaire ${ }^{10}$

Visa permettant à l'étranger spécifiquement soumis à cette exigence, de passer par la zone internationale de transit d'un aéroport et ce, sans accéder au territoire national du pays concerné, lors d'une escale ou d'un transfert entre deux tronçons d'un vol international. L'exigence de ce visa est une exception au privilège général de transit sans visas par ladite zone internationale de transit.

2.1.2 Visa autorisant un étranger qui se rend d'un Etat tiers vers un autre Etat tiers à traverser le territoire des Parties contractantes ou visa de transit.

Ce visa peut être délivré pour un, deux ou, exceptionnellement, plusieurs transits, sans pour autant que la durée de chaque transit puisse excéder cinq jours.

2.1.3 Visa de court séjour ou de voyage ; visa à entrées multiples

Visa permettant à un étranger de solliciter l'entrée sur le territoire des Parties contractantes pour des motifs autres que l'immigration, en vue d'un séjour ininterrompu ou de plusieurs séjours dont la durée totale ne dépasse pas trois mois par semestre à partir de la date de première entrée. Ce visa peut être délivré, en règle générale, pour une ou plusieurs entrées.

Dans le cas de certains étrangers qui doivent se rendre fréquemment dans un ou plusieurs Etats Schengen dans le cadre par exemple de voyage d'affaires, le visa pour un séjour de courte durée peut être délivré pour des séjours multiples, la durée totale de ces séjours ne pouvant excéder trois mois par semestre. La validité de ce visa multiple peut être d'un an et, exceptionnellement, de plus d'un an pour certaines catégories de personnes » 
D'après ce dernier passage, il est évident qu'il y a des personnes qui échappent complètement à la définition de leur nationalité comme risque pour l'Union. Mais on verra plus tard que ces personnes sont définies par leur puissance économique.

«2.1.4 Visa collectif

Visa de transit ou d'une durée ne dépassant pas trente jours, qui peut être apposé sur un passeport collectif - sauf si la législation nationale en dispose autrement - délivré à un groupe d'étrangers, constitué préalablement à la décision d'entreprendre le voyage, et à condition que les membres du groupe entrent sur le territoire, y séjournent et le quittent en tant que groupe.

Le visa collectif est délivré pour des groupes composés d'un nombre de personnes compris entre 5 et 50 personnes. La responsable du groupe devra disposer d'un passeport individuel et, si nécessaire, d'un visa individuel ».

Ici, l'aspect réseau du visa Schengen est particulièrement clair. La législation nationale est à même de reconnaître ou non un type de visa Schengen - le visa collectif. Elle est ainsi une norme supérieure à la mesure d'harmonisation. Il en découle que le visa collectif uniforme n'a donc pas la même valeur dans les différents Etats puisque l'on peut être stoppé à la frontière de l'Etat qui ne le reconnait pas. La notion d' uniformité débouchant sur un espace territorial unique est ici plus que virtuelle.

Par ailleurs, aux cinq catégories de visas Schengen s'ajoutent trois autres types de visas nationaux, les visas de long séjour, les visas à validité territoriale limitée et les visas délivrés a la frontière, qui peuvent dans certains cas faire office d'équivalent fonctionnel du visa Schengen. Concernant les visas de long séjour, la Commission écrit : «Le visa pour un séjour supérieur à trois mois est un visa délivré par chaque Partie contractante conformément à sa propre législation.

Toutefois, il aura valeur de visa uniforme de transit permettant à son titulaire de se rendre sur le territoire de la Partie contractante de délivrance du visa, étant entendu que la durée du transit n'excède pas cinq jours à compter de la date d'entrée, sauf si le titulaire ne réunit pas les conditions d'entrée ou s'il est signalé aux fins de nonadmission par la ou les Parties contractantes dont il souhaite traverser le territoire ».

Concernant les deux autres formes de visas («Visa à validité territoriale limitée et visa délivré à la frontière »),les modalités de ces derniers se trouvaient dans les instructions aux frontières qui étaient confidentielles et qui, depuis peu, sur l'insistance de quelques ONG dont Statewatch, ont été déclassifiées et publiées dans le manuel commun ${ }^{11}$. Les visas à validité territoriale limitée sont délivrés par une des parties contractantes " pour des motifs humanitaires ou d'intérêt national ou en raison d'obligations internationales " lorsqu'elle estime que les conditions prévues pour le visa uniforme ne s'appliquent pas mais veut néanmoins délivrer un visa. Il en va de même si l'Etat veut délivrer un nouveau visa au cours du même semestre. Ces visas permettent de traverser mais pas de séjourner sur le territoire des autres parties contractantes.

Comme on le voit la dérogation permettant à un Etat de faire ce qu'il désire a toujours existé, même si on le découvre simplement dans ce texte rendu public en $2002^{12}$.

Les visas délivrés à la frontière le sont, s'il apparait qu'il existe des motifs impérieux et un manque de temps mais il faut que l'étranger soit titulaire d'un document valable permettant le franchissement de la frontière, qu'il remplisse les conditions prévues à l'article $5 \$ 1$ a) b) c) d) e) de la Convention d'application et que son retour dans son pays ou son transit vers un Etat tiers soit garanti. Ces visas pourraient être utiles aux 
demandeurs d'asile fuyant les conflits mais il semble que leur usage soit limité dans ce cas, bien qu'il existe, et qu'on voit parfois un usage de ces visas pour des personnels engagés dans des actions que l'on appellera d'anti-diplomatie ${ }^{13}$.

$\mathrm{Au}$ total, le visa Schengen dit uniforme n'est donc pas un visa unique. Il comprend différents types de visas, correspondant à des modalités de voyage ou de séjour différentes, ce qui est normal. Mais l'applicabilité territoriale est sujette à variation selon le bon vouloir des Etats ce qui est plus problématique. De plus, le visa Schengen ne s'impose pas automatiquement aux Etats Schengen. Ils le reconnaissent ou non selon les modalités qui varient d'un pays à l'autre. Enfin, même lorsque l'on pense avoir atteint une certaine harmonisation, on s'aperçoit que les disparités $d$ 'applications sont fondamentales.

Le prix uniformisé du visa?

Parmi les modalités dites uniformes, il y a le prix du visa. Pendant plusieurs années le montant des droits à percevoir correspondant aux frais administratifs de traitement de la demande de visa a varié selon les consulats nationaux dans un rapport de un à huit. Maintenant, avec l'euro, chaque consulat se fait payer un même montant. Néanmoins, les droits à percevoir liés à la délivrance du visa uniforme varient selon le type de visa comme le montre le tableau suivant permettant de suivre en détail les différents types de visas ${ }^{14}$ :

A. Transit aéroportuaire 10 EUROS

Transit

(une, deux ou plusieurs entrées) 10 EUROS

C1. Très courte durée (30 jours) 30 EUROS + 5 EUROS à partir de la 2 e entrée en cas d'entrées multiples

C2. Courte durée (90 jours max.) 50 EUROS

C3. Entrées multiples, durée de validité d'un an 50 EUROS

C4. Entrées multiples, durée de validité de 5 ans max. 50 EUROS + 30 EUROS par année supplémentaire

D. visa national pour un long séjour Montant fixe par les Parties contractantes, ces visas pouvant être gratuits

- validité territoriale limitée Montant au moins égal a 50\% du montant fixe pour les visas de catégories $\mathrm{A}, \mathrm{B}$ ou $\mathrm{C}$

- délivré à la frontière Montant double de celui correspondant à la catégorie de visa délivré. Ces visas peuvent être gratuits

Collectif, catégories A \& B (de 5-50 personnes) 10 EUROS + 1 EURO par personne

Collectif, catégorie C1 (30 jours) 1 ou 2 entrées (de 5-50 personnes) 30 EUROS + 1 EURO par personne

Collectif, catégorie C1 (30 jours) plus de deux entrées (de 5-50 personnes) 30 EUROS + 3 EUROS par personne

Ces prix ont été fixés, après un débat, où s'opposaient d'un côté ceux qui voulaient en faire une arme dissuasive contre l'immigration et les voyages trop faciles vers l'Europe et ceux qui voulaient faciliter la libre circulation. Ces derniers ont plutôt obtenu gain de cause. Le prix est resté modéré. 
Mais, à ce prix officiel, il ne faut pas oublier d'ajouter pour les individus le prix du passeport lui-même et surtout le prix des documents certifiés qu'on leur demande pour obtenir un visa et qui sont parfois coûteux - relevés bancaires sur deux ans facturés par la banque par exemple ${ }^{15}$. Il faudra aussi que les candidats au visa fassent la preuve qu'ils disposent d'une somme qui varie d 'un pays à l'autre (environ 40 euros) par jour pour la durée de leur séjour et d'un billet de retour. Certains consulats exigent de voir l'argent avant le départ alors que d'autres se contentent d'une déclaration signée. Il leur faut aussi prouver que sans travailler, ils ont les moyens de se loger, soit à l'hôtel, soit chez des particuliers qui les reçoivent. Or, ici, chaque législation nationale reprend ses droits et fixe des règles différentes quant au séjour, fut-il de moins de trois mois. L'annexe 7 de l'ICC récapitule toutes ces différences ${ }^{16}$.

Ainsi, les règles qui régissent la question de la responsabilité des Etats pour considérer une demande de visa sont admirablement compliquées car elles entrent en interaction avec les lois sur l'immigration clandestine et le séjour des étrangers qui restent du domaine national et ne sont pas uniformisées. Virginie Guiraudon a donné plusieurs exemples des différences existant entre un individu d'un pays-tiers qui veut se rendre en France, en Allemagne ou aux Pays-Bas, bien qu'il y ait visa uniforme, s'il lui faut par exemple être hébergé chez un national ; les modalités pratiques d'accueil des étrangers variant fortement d'un pays à l'autre, même si la tendance générale est celle d'un renforcement des contrôles portés sur les nationaux hébergeant des étrangers.

Parler de visa uniforme Schengen est donc un abus de langage. Parler de régime migratoire européen est encore plus discutable. Partout les logiques de réseau l'emportent sur celles de l'harmonisation et de l'unification à long terme, partout les disjonctions sont plus fortes que les conjonctions, et quand ces dernières existent, elles se font sur la base de technologies identiques dans la police à distance et autour d'une idéologie de la suspicion et du contrôle migratoire, non pas autour de l'idée de libre circulation ou de celle de favoriser la vitesse de circulation des personnes comme c'était le cas il y a vingt ans. La notion foucaldienne de « dispositif » est sans doute celle qui rend le mieux compte de ces pratiques hétérogènes mais mises en série comme on va s'en apercevoir en étudiant de plus près les critères par lesquels on place un pays dans la liste des pays soumis à visa ou exempté de visa, de même que par l'analyse des critères qui débouchent sur l'attribution ou non individuelle d'un visa quand celui-ci est obligatoire pour se rendre sur le territoire Schengen.

\section{NOTES}

1.. Voir la plaquette de présentation en annexe (Cultures \& Conflits, $\mathrm{n}^{\circ} 50$, Eté 2003).

2. . JOCE du 16 Décembre 2002 C 313, voir annexe (Cultures \& Conflits, n50, Eté 2003).

3. . Entretiens à la Commission. Février 2001.

4. . Discussion avec des responsables nationaux et de la Commission. Voir aussi l'annexe sur le modèle uniforme de titre de séjour du 13/06/2002 (Cultures \& Conflits, n50, Eté 2003). 
5. . Part I, paragraphe 2.1 de l'instruction consulaire commune définissant le visa. JOCE C 313, p. 6 (site C\&C).

6. . Certaines $O N G$ ont fait remarquer que si l'État dans lequel on veut séjourner le plus longtemps, refuse de se déclarer compétent en considérant qu'il n'est pas l'État de destination principale, et en désigne un autre, qui, lui-même, ne veut pas non plus regarder la demande de visa, on a des situations de « mise en orbite » où chaque consulat se renvoie la « balle ». Nous sommes loin du shopping. Quant aux situations de shopping, elles sont dépendantes des agents des consulats qui soit considèrent les politiques comme trop dures et font de la résistance au quotidien en aidant les demandeurs, soit se font payer leurs services comme les consulats de France et de Belgique en Bulgarie.

7.. Il est de plus en plus difficile si l'on n'est pas un homme d'affaires connu de demander un visa depuis son consulat dans un pays tiers. Il faut revenir dans son pays d'origine pour demander le visa, ce qui peut être totalement dissuasif. Par exemple, pour un Sud-africain qui visite le Royaume-Uni et voudrait venir pour une journée en France.

8. . Les agents consulaires et certains responsables des ministères des Affaires étrangères des divers pays Schengen sont souvent eux-mêmes mal renseignés, confusion entre Schengen et l'Union, incompréhension de la sphère de collaboration avec le Royaume-Uni, confusion sur les types de visas.

9. . Voir dans les dispositions générales, la définition du visa uniforme art 2.1 et les différents types de visas JOCE C313, p. 6.

10. . Voir annexe sur le visa de transit aéroportuaire (Cultures \& Conflits, $n^{\circ} 50$, Eté 2003).

11. . Manuel Commun, C313-02 JOCE C313, p. 102 et p. 114 (site de C\&C).

12. . Heiner Busch l'avait signalé depuis 1995 dans ses articles. Voir le numéro de son article « Le piège légaliste. La coopération policière après Tampere ", Cultures \& Conflits, De Tampere à Séville : bilan de la sécurité européenne (1), nº5, Paris, L'Harmattan, 2002.

13. . Sur les pratiques d'anti-diplomatie ou d'espionage voir l'ouvrage de James der Derian, Antidiplomacy, spies, terror, speed, and war, Blackwell, Oxford, 1992, IX-215 p. La protection aux frontières ne va pas forcément à ceux qui en ont le plus besoin.

14. . Annexe 12 des ICC (JOCE C313, p. 65).

15. . En Côte d'Ivoire par exemple un tel relevé peut être facturé par la banque à l'équivalent d'un mois de salaire.

16. . Annexe 7 des ICC (JOCE C313, pp. 54-55). Cf. Cultures \& Conflits, n50, Eté 2003.

INDEX

Index géographique : Schengen

Mots-clés : coopération internationale, réseaux transnationaux, Visa

Thèmes : Schengen (convention) 